\title{
A CMOS Frequency divider for 2.4/5GHz WLAN Applications with a Simplified Structure
}

\author{
Q. Yu*, Y. Liu*, X.P. Yu**, W. M. Lim**, F. Yang*, X. L. Zhang*, and Y. Peng*
}

\begin{abstract}
In this paper, a dual-band integer- $N$ frequency divider is proposed for $2.4 / 5.2 \mathrm{GHz}$ multistandard wireless local area networks. It consists of a multi-modulus imbalance phase switching prescaler and two all-stage programmable counters. It is able to provide dual-band operation with high resolution while maintaining a low power consumption. This frequency divider is integrated with a $5 \mathrm{GHz}$ VCO for multi-standard applications. Measurement results show that the VCO with frequency divider can work at $5.2 \mathrm{GHz}$ with a total power consumption of $22 \mathrm{~mW}$.
\end{abstract}

Index Terms-Frequency divider, frequency synthesizer, phases switching, wireless local area network (WLAN)

\section{INTRODUCTION}

The wireless communication industry is currently experiencing a tremendous growth. For Wireless LAN applications, the IEEE 802.11 multiple standards have been widely adopted for the short-range communication $[1,2]$. The frequency synthesizer, which is usually formed by a Phase-Locked Loop (PLL), is a major and critical component of a wireless transceiver because it operates at high frequency and consumes a large portion of the total power in the transceiver. Currently, there exist

\footnotetext{
Manuscript received Jul. 19, 2011; revised Oct. 15, 2011.

* State Key Laboratory of Electronic Thin Films and Integrated Devices, University of Electronic Science and Technology of China, Chengdu 610054, P. R. China

** School of Electrical and Electronic Engineering, Nanyang Technological University, Singapore 639798

E-mail : yliu2008@e.ntu.edu.sg,xpyu@pmail.ntu.edu.sg
}

several different standards of operation, for example, the 2.4 GHz 802.11 b/g and 5.2 GHz 802.11a/ HIPERLAN-2 networks; and thus a multi-standard frequency synthesizer is desirable for operations under different wireless systems. Numerous research efforts have been carried out on multi-band multi-standard receivers [3-9]. The performance in power consumption and channel selection of a frequency synthesizer are limited by the two important building blocks, namely the frequency divider and voltage-controlled oscillator (VCO). The fractional- $N$ frequency synthesizer has been used to achieve high resolution [2]. However, it has problem of spurs and a complex circuit, e.g. the sigma-delta modulator is needed to suppress spurs [2, 10]. On the other hand, the integer- $N$ frequency synthesizer takes the advantage of low-cost and low-spur sideband performance [10]. The frequency divider in the integer- $N$ frequency synthesizer is usually formed by a prescaler, a program counter ( $P$ counter) and a swallow counter $(S$ counter) [11] which can provide a programmable division ratio of $N \times P+S$, where $N, P$ and $S$ are the division ratios of the prescaler, $P$ counter and $S$ counter, respectively. In the conventional designs [1], dual-loop or triple loop frequency synthesizers, additional mixers are used to obtain desired frequencies. This requires complex topologies and introduces additional power consumptions. In this paper, a wide-band high-resolution programmable frequency divider with a simple topology is proposed. This paper is organized as follows. In section II, the design considerations for the frequency synthesizer are reviewed. Section III describes the structure and operation of the proposed circuits. The experimental results are summarized in section IV. Finally, the conclusions are given in Section V. 


\section{DESIGN CONSIDERATIONS}

The considerations in the frequency synthesizers design include: high operating frequency, low power consumption, and low phase noise. High operation frequency and low power consumption are key considerations in the frequency divider design. Injection locked frequency divider has been proposed to achieve high operation frequency and low power consumption [10]. However, it is with limited bandwidth and hard to achieve variable division ratios [10]. Therefore, in the applications of multi-channel WLANs, the digital frequency divider is commonly used. The operating frequency of digital frequency divider is inversely proportional to the propagation delay while digital building blocks in high frequencies take major contribution to the total power consumption because the power consumption is usually linearly proportional to the operating frequency. In the multi-standard applications, there are some additional considerations: as the frequency synthesizer is used for multiple standards, all the carrier frequencies of these standards must be produced. The VCO and the frequency divider must be adapted to provide the desired frequencies for downconversion and up-conversion in the transceiver. The major challenge lies in the frequency divider design, which must provide a wide range and a high resolution for the frequency divisions. To save area and power consumption, a simple topology is preferred.

\section{CirCUIT ImPLEMENTATIONS}

A simple method to achieve the $2.4 / 5.2 \mathrm{GHz}$ dualband operation can be realized by utilizing a $5 \mathrm{GHz}$ oscillator for the $5.2 \mathrm{GHz}$ wireless LAN and a divide-by2 unit to provide $2.4 \mathrm{GHz}$ frequency band. With this method most of the circuits for dual-band operation can be shared. Considering the gap between the oscillator (2.6 GHz) of the divide-by- 2 and the $2.4 \mathrm{GHz}$ wireless LAN applications, a wide operating range is required for both the oscillator and the frequency divider.

\section{Wide band Voltage-Controlled Oscillator}

The voltage controlled oscillator (VCO) is a key building block in the design of a frequency synthesizer.

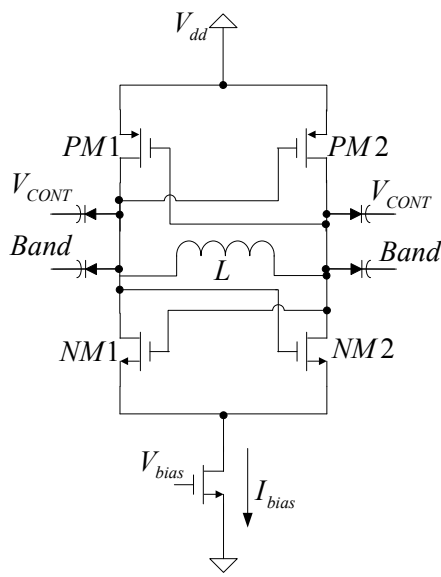

Fig. 1. The topology of the proposed VCO.

The complementary cross-coupled structure which is suitable for high frequencies of oscillation is used, as shown in Fig. 1.

It utilizes NMOS and PMOS cross-coupled amplifiers as the negative resistance to compensate for the losses in the LC tank. The resonant tank includes the parasitic capacitances of MOS transistors and of the inductor. Frequency tuning is realized by control of back gate voltages of the varactors. Two groups of varactors, which are implemented with $\mathrm{PN}$ varactor, are controlled with two external DC voltage sources, namely Band and $V_{\text {CONT }}$. The size of the varactors is $100 \mathrm{fF}$. The tuning range of the $\mathrm{VCO}$ is around $15 \%$, from $4.7 \mathrm{GHz}$ to 5.3 GHz. Larger varactors could be used to achieve a wider tuning range by adjusting the transistor size accordingly. In the operating range of $5 \mathrm{GHz}$, the performance of phase noise is mainly determined by the quality factor of the inductor. The size of the inductor is carefully optimized to obtain a higher quality factor.

\section{Dual-band frequency divider}

A high resolution is required to cover all the center frequencies of multi-standards applications, as the frequency synthesizer is based on the integer- $N$.

This demands a low-power and high-resolution frequency divider. The most challenging block in the design of a high frequency programmable divider is the prescaler. In conventional designs [1, 10], a divide-by-2 unit is placed before the prescaler, making all the division ratio in the order of 2 . The method sets a limitation in the available division ratios. The phase 
switching technique, as proposed in [12] can be implemented to solve this problem.

Fig. 2 shows the topology of a phase switching prescaler [12]. The phase switching prescaler has many advantages over the conventional prescaler based on the Johnson cell [12]. The phase switching prescaler can be implemented with only one full speed divide-by-2 unit. However, the conventional prescaler normally utilizes a divide-by- $2 / 3$ or $4 / 5$ unit, which has additional power consumption and propagation delay. Moreover, the dualmodulus operation by phase switching makes this topology suitable for large division ratios and multi-band operations. In the conventional design, an end-ofcounting detector is employed to detect all divide-by-2 stages' output. The logic detector becomes complex with the increase of number of the divide-by-2 stages. This creates difficulties in the dual-band operations as the detector is used to detect all stages' status. For the phase switching prescaler, the change of modulus is controlled by switching between different phases at the ending of a division cycle. Only a 2-to-1 MUX in the divide-by-2 chain is required when changing from divide-by- $N / N+1$ to divide-by- $2 N / 2 N+1$. This topology makes it easy to perform dual-band operations.

As shown in Fig. 3, a 2-to-1 MUX is used in the divide-by-2 chain to select different divide-by-2 stage as the output, which is similar to the multi-modulus divider in [13]. To increase the operating frequency, the imbalanced phase switching, which can switch between $25 \%$ duty cycle phase signals instead of $50 \%$ duty cycle signals, is used [14]. This prescaler can provide dualband control in additional to the dual-modulus operation. The merit of the proposed design is that, the output of the prescaler is divide-by-N/N+1 and divide-by- $2 \mathrm{~N} / 2 \mathrm{~N}+1$. In the conventional design with a divide-by-2 in the oscillator, the output of the oscillator and divide-by-2 unit are divide-by- $\mathrm{N} /(\mathrm{N}+1)$ and divide-by- $2 \mathrm{~N} /(2 \mathrm{~N}+2)$. The proposed design is able be integrated with the pulse-

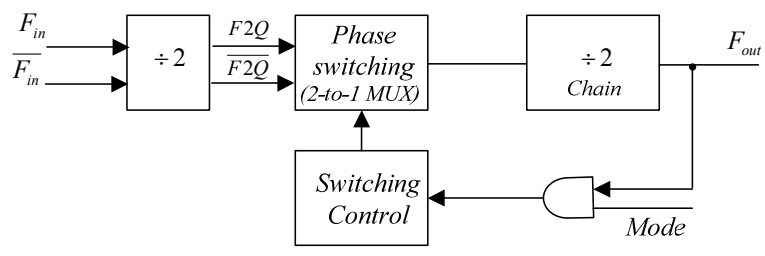

Fig. 2. The topology of 4-to-1 phase switching prescaler.

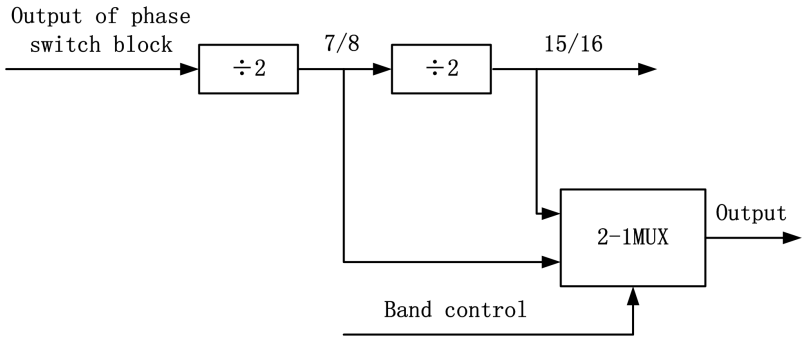

Fig. 3. The proposed dual-band operation in the prescaler.

swallow counters to provide programmable division ratios easily. The total division ratio of the proposed frequency divider will be $N \times P+S$ and $2 N \times P+S$. In the conventional design, the ratio is $N \times P+S$ and $2(N \times P+S)$ which are limited to the multiple of 2 .

In a wide-range high-resolution operation, the configuration of the digital counter plays an important role. In an integer- $N$ frequency synthesizer, the total division ratio is given by $N \times P+S$. In the conventional design, only the $\mathrm{S}$ counter is partially programmable [10] If all stages of both $P$ and $S$ counters are programmable, the total division ratios available will be increased. To achieve high-resolution, wide-band operation with a low power consumption, the digital counter proposed in the [11] is used in this frequency divider.

\section{EXPERIMENTAL RESULTS}

The proposed circuits are fabricated using the Chartered Semiconductor Manufacturing (CSM) $0.18 \mu \mathrm{m}$ $1 \mathrm{P} 6 \mathrm{M}$ CMOS process. Measurement is carried out on wafer by using a Cascade probe station. The output of the VCO is measured by Agilent $26.5 \mathrm{GHz}$ spectrum analyzer. In the VCO's test chip, common-source buffers with $50 \mathrm{ohm}$ resistive loads are used. Due to parasitic capacitors in the NMOS of the buffers, the output frequency reduces slightly. Fig. 4 shows the output spectrum of the $\mathrm{VCO}$, and its operating range is summarized in Fig. 5. The phase noise of the VCO at $5 \mathrm{GHz}$ is presented in Fig. 6 . At the offset of $1 \mathrm{MHz}$, the phase noise is about $-105 \mathrm{dBc} / \mathrm{Hz}$.

The dual-band prescaler and the frequency divider are integrated with the frequency divider combining with the VCO. Post-layout simulation results show the prescaler implemented with the proposed 2-to-1 phase switching technique is able to work at a input frequency of $5.2 \mathrm{GHz}$ Due to the lack of differential input signal for the 
proposed measurements, a transgate and an inverter are placed in the input signal for measurement. The mismatch caused by the input slightly reduces the operating frequency.

The prescaler is implemented with the Giga-Hertz

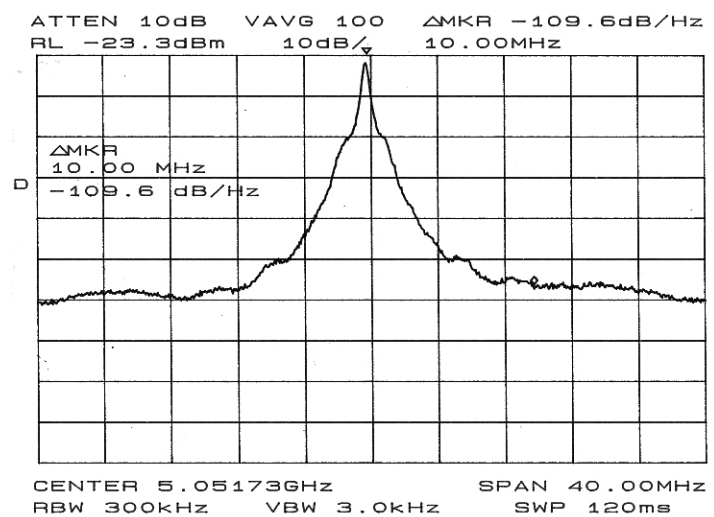

Fig. 4. Output spectrum of the proposed VCO.

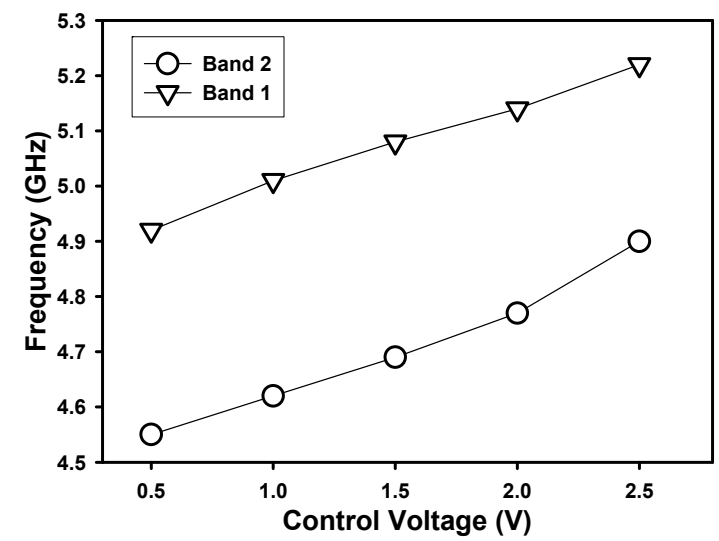

Fig. 5. The tuning range of the proposed VCO.

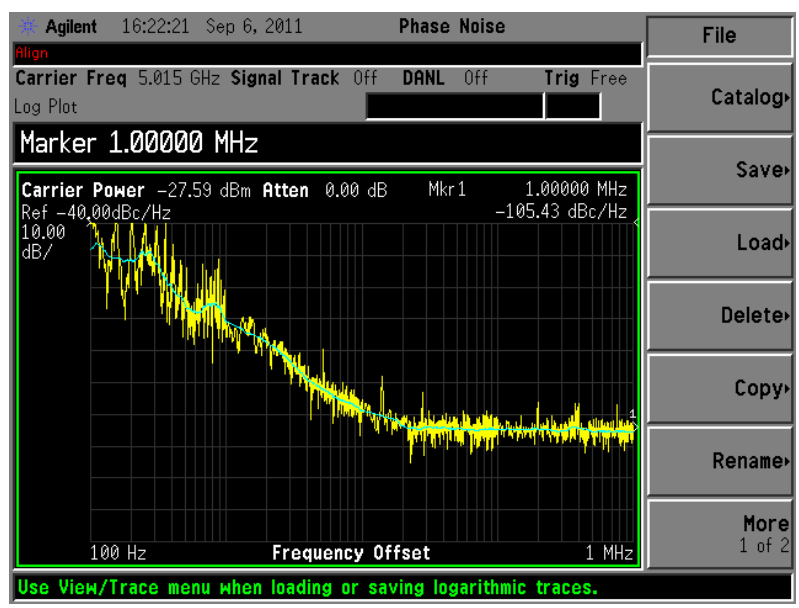

Fig. 6. Phase noise spectrum of the proposed VCO. range digital counter [11] to realize a wide range high resolution frequency divider. The single-ended input source is provided by the Antristu $68347 \mathrm{C} 10 \mathrm{MHz}-20$ $\mathrm{GHz}$ signal generator. The output signals are captured by Lecroy Wavemaster 8600A 6G oscilloscope. The stand alone frequency divider can operate at a input frequency of $4.5 \mathrm{GHz}$. Fig. 7 shows the output waveform of the prescaler at an input frequency of $4 \mathrm{GHz}$ having dualmodulus and dual-band operation. Fig. 8 shows the operating frequency vs. power consumption for the proposed frequency divider which is implemented with this prescaler and two counters in [11]. Here the division ratio of the proposed frequency divider is set as 1171 to demonstrate the programmable division ratio. The covering of different WLAN standards can be achieved by changing the configuration of $\mathrm{N}, \mathrm{P}$ and $\mathrm{S}$. The power consumptions for the VCO and frequency divider are 7 $\mathrm{mW}$ and $15 \mathrm{~mW}$, respectively. Fig. 9 shows the die photo of the proposed VCO with dual-band frequency divider.
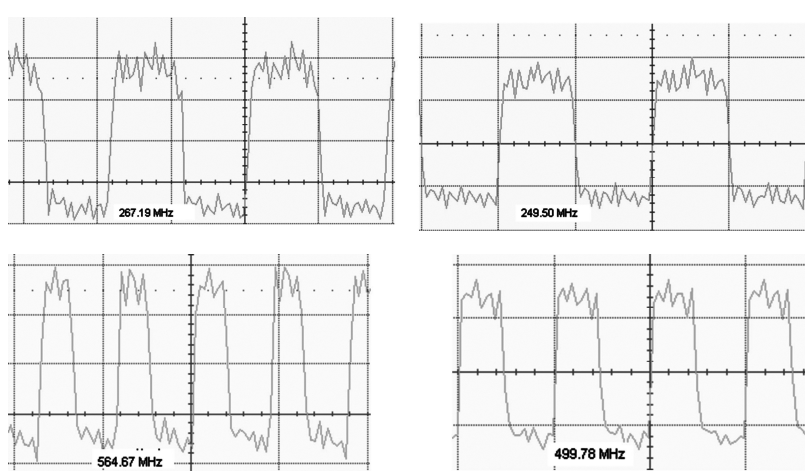

Fig. 7. Measured output waveform of the proposed prescaler with divide-by-7/8/15/16 operation.

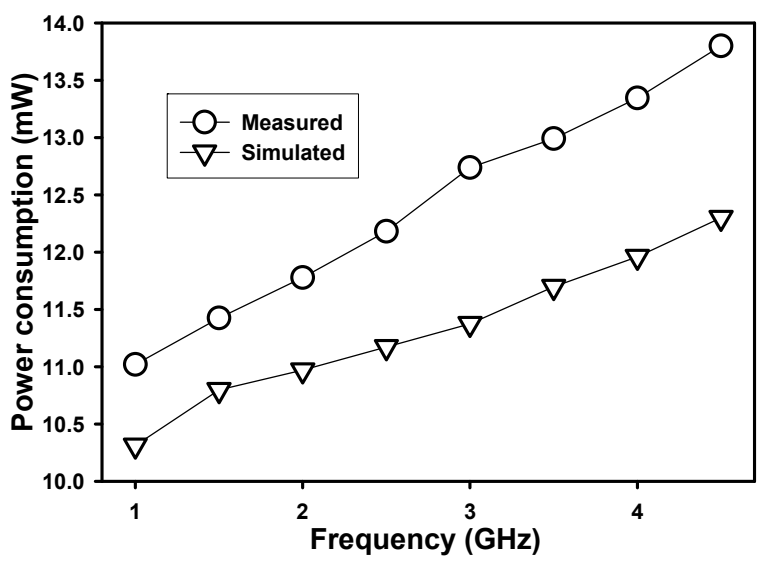

Fig. 8. Power consumption within operation range. 
Table 1. Summary of the works

\begin{tabular}{|c|c|c|c|c|c|}
\hline Parameters & {$[3]$} & {$[6]$} & {$[8]$} & {$[15]$} & This work \\
\hline Process (CMOS) & $0.24 \mu \mathrm{m}$ & $0.18 \mu \mathrm{m}$ & $0.18 \mu \mathrm{m}$ & $0.18 \mathrm{um}$ & $0.18 \mu \mathrm{m}$ \\
\hline Supply Voltage & $1.5 \mathrm{~V}$ & $1.8 \mathrm{~V}$ & $1.8 \mathrm{~V}$ & $1.8 \mathrm{~V}$ & $1.8 \mathrm{~V}$ \\
\hline Frequency & $5 \mathrm{GHz}(1 \mathrm{GHz}$ range $)$ & $6.3-8.9 \mathrm{GHz}$ & $\mathrm{N} / \mathrm{A}$ & $5.035-5.435 \mathrm{GHz}$ & $1-5.2 \mathrm{GHz}$ \\
\hline Division ratios & $2 \times(220-227)$ & $13-20$ & $\mathrm{~N}=8^{*}(150-\mathrm{S})+(8+0.5) * \mathrm{~S}$. & $5 \times(259-266)$ & $\begin{array}{c}7 / 15 \times P+S \\
P, S \text { are } 2-2^{\mathrm{n}}(\mathrm{P}>\mathrm{S})\end{array}$ \\
\hline Power consumption & $19 \mathrm{~mW}$ & $31.5 \mathrm{~mW}$ & $10 \mathrm{~mW}$ & about $15 \mathrm{~mW}$ & $15 \mathrm{~mW}$ \\
\hline $\begin{array}{c}\text { F. O. M. } \\
\text { (freq*bandwidth/Power) }(\mathrm{GHz})^{2} / \mathrm{mW}\end{array}$ & 0.27 & 0.6 & N/A & 0.139 & 0.868 \\
\hline
\end{tabular}

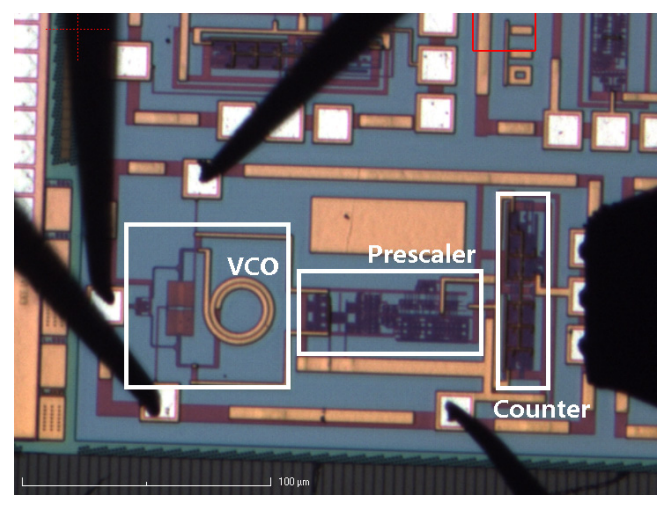

Fig. 9. Die photo of the proposed VCO with frequency divider.

Function blocks including VCO, prescaler and counter are marked in the figure. Table 1 presents the result from this work and those from other dividers in references in terms of power consumption, operating frequency, availability of the division ratios and F.O.M. F.O.M. is defined as operating frequency times bandwidth over power consumption.

\section{Conclusions}

In this paper, a multi-standard WLAN frequency synthesizer is proposed, including a VCO with a wide tuning range, a dual-band dual-modulus phase switching prescaler and Giga $\mathrm{Hz}$ range digital counters. The VCO and frequency divider is able to cover the application of 5.2/2.4 GHz wireless LAN operations with low power consumption. The performance of the $\mathrm{VCO}$ and the frequency divider are verified with a CMOS technology.

\section{ACKNOWLEDGMENT}

This work has been financially supported by NSFC under project No.60806040, the Fundamental Research Funds for the Central Universities under project
No.ZYGX2009X006, and the Young Scholar Fund of Sichuan under project No.2011JQ0002.

\section{REFERENCES}

[1] Chang, S.-F.R., et al, "A dual-band RF transceiver for multistandard WLAN applications," IEEE Trans. Microwave Theory \& Tech., Vol.53, No.3, pp.1048-1055, 2005.

[2] Pengfei Zhang, et al, "A single-chip dual-band direct-conversion IEEE $802.11 \mathrm{a} / \mathrm{b} / \mathrm{g} \quad$ WLAN transceiver in 0.18- $\mu \mathrm{m}$ CMOS," IEEE J. SolidState Circuits, Vol.40, No.9, pp.1932-1939, 2005.

[3] Adiseno, A., et al, "A 3-V area-efficient wideband low-noise converter for multi-band multi-standard low-IF wireless receiver," Solid-State Circuits Conference, 2002. ESSCIRC 2002. Proceedings of the 28th European, art. No.186889, pp.791-794, 2002.

[4] Chao-Shiun Wang, et al, "A multi-band multistandard RF front-end IEEE 802.16a for IEEE 802.16a and IEEE 802.11 a/b/g applications.," Proceedings - IEEE International Symposium on Circuits and Systems, No.1465501, pp.3974-3977, May, 2005.

[5] Rivet, F., et al, "A universal radio frequency receiver architecture based on sampled analog signal processing," 2007 IEEE North-East Workshop on Circuits and Systems, NEWCAS 2007, art. No.4488024, pp.1449-1452, Aug., 2007.

[6] Behjou, N., Larsen, T., Hoegdal, M., "Design of a simultaneous multi-band RF sub-sampling receiver," IEEE MTT-S International Microwave Symposium Digest, art. No.4633089, pp.5-8, 2008.

[7] Mustaffa, M.T., Zayegh, A., Zulkifli, T.Z.A., “A reconfigurable LNA for multi-standard receiver using $0.18 \mu \mathrm{m}$ CMOS technology," SCOReD2009 - 
Proceedings of 2009 IEEE Student Conference on Research and Development, art. No.5443076, pp.238-241, Nov., 2009.

[8] Huijung Kim, et al, "A multi-standard multi-band tuner for mobile TV SoC with GSM Interoperability," Digest of Papers - IEEE Radio Frequency Integrated Circuits Symposium, art. No.10.1109/RFIC.2010.5477290, pp.189-192, May, 2010.

[9] Vazny, R., et al, "An interstage filter-free mobile radio receiver with integrated TX leakage filtering," Digest of Papers - IEEE Radio Frequency Integrated Circuits Symposium, art. No.10.1109/ RFIC.2010.5477294, pp.21-24, May, 2010.

[10] H.R. Rategh, H. Samavati, T.H. Lee, "A CMOS frequency synthesizer with an injection-locked frequency divider for a 5-GHz wireless LAN receiver," IEEE J. Solid-State Circuits, Vol.35, No.5, pp.780-787, May 2000.

[11] Yu, X.P., et al, "Design of a low power wide-band high resolution programmable frequency divider.," IEEE trans. VLSI, Vol.13, No.9, pp.1098-1103, Sep., 2005.

[12] Keliu Shu, et al, "A 2.4-GHz monolithic fractional$\mathrm{N}$ frequency synthesizer with robust phaseswitching prescaler and loop capacitance multiplier," IEEE J. Solid-State Circuits, Vol.38, No.6, pp.866-874, Jun., 2003.

[13] Geum-Young Tak, et al, "A 6.3-9-GHz CMOS fast settling PLL for MB-OFDM UWB applications," IEEE J. Solid-State Circuits, Vol.40, No.8, pp.1671-1679, Aug., 2005.

[14] Yu, X.P., et al, "Low power high-speed CMOS dual-modulus prescaler design with imbalanced phase-switching technique," IEEE proc. Circuits, Devices and Systems, Vol.152, No.2, pp.127-132, Apr., 2005.

[15] Ping-Yuan Deng, and Jean-Fu Kiang, "A 5-GHz CMOS Frequency Synthesizer with an injectionlocked frequency divider and differential switched capacitors," IEEE Trans. Circuit and Systems-I, Vol.56, No.2, pp.320-326, Feb., 2009.

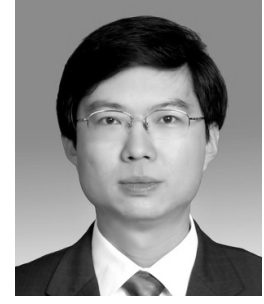

Q. Yu was born in YanTai, Shandong Province of China in 1972. $\mathrm{He}$ received Master and $\mathrm{PhD}$ Degree at University of Electronic Science and Technology of China in 1997 and 2010, respectively. In 2011, he was promoted to be full professor. Sponsored by China International Talent Exchange Foundation, he had been at IMEC for the integrated circuits design program for three months in 2007. His main research interests focus on novel semiconductor devices and mixed signal integrated circuits design. From 1997 to present, he has accomplished more than 20 research projects in the field of semiconductor devices and integrated circuits. He has filed five China patents and has published 30 papers in international conferences and journals. He was awarded the prestigious Award of Science and Technology of China.

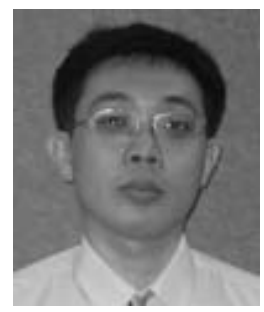

Y. Liu received the B.Sc. degree in microelectronics from Jilin University, China, in 1998 and the Ph.D. degree from Nanyang Technological University, Singapore, in 2005. From May 2005 to July 2006, he was a Research Fellow with Nanyang Technological University, Singapore. In 2006, he was awarded the prestigious Singapore Millennium Foundation Fellowship. In 2008, he joined the School of Microelectronics, University of Electronic Science and Technology, China, as a Full Professor. He is the author or coauthor of 80 peer-reviewed journal papers and more than 30 conference papers. His current research includes nanoscale CMOS device reliability, RF/MM ICs and photonic/optoelectronic devices and ICs. 


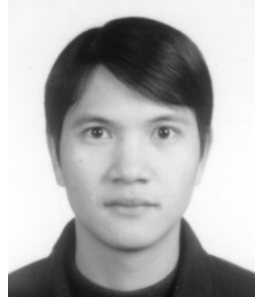

X.P. Yu was born in Zhejiang, P.R. China. He received the B.Eng. degree in 1998 from the Department of Optical Engineering, the Information College, Zhejiang Univ., Yu Quan, Hangzhou, P.R. China, and the PHD degree from School of Electrical \&

Electronic Engineering, Nanyang Tech. Univ., Singapore, in 2006. Before joined NTU as a PHD candidate in 2002, he has been with MOTOROLA Global Telecom Solution Sector, Hangzhou, P.R. China. Since Sep. 2005, He has been a research staff in NTU. He joined the Institute of VLSI Design, Zhejiang Univ., on Sep. 2006 as a lecturer. $\mathrm{He}$ is now Associate Professor. Since Jan. 2008, he has been with Eindhoven Univ. of Tech., the Netherlands as a visiting scholar. While from Aug. 2009, he has been a Marie Curie Fellow in this group (Co-hosted with Philips Research, Eindhoven). His research interests include CMOS radio frequency integrated circuits for wireless communication, low-power phase-locked loops and clock data recovery circuits for high-speed data communications using submicron CMOS technology.

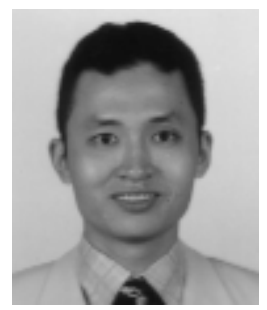

W. M. Lim received his B.E (with honors) and M.E degrees from Nanyang Technology University (NTU), Singapore in 2002 and 2004 respectively. Upon his graduation, he joined the School of Electrical and Electronic Engineering, NTU, as a research staff. His research interests include RF circuit designs, RF devices characterization, and modeling.

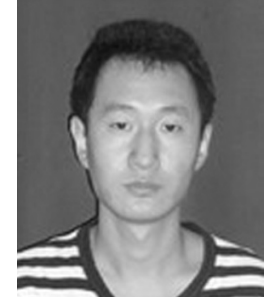

F. Yang was born in Jilin, China, in 1988. He received the bachelor degree in microelectronics from University of Electronic Science and Technology, China, in 2010. He is presently pursuing a master's degree at the UESTC. He is engaged in CMOS Analog/RF integrated circuit design mainly for RF-transmitter and RF-receiver applications.

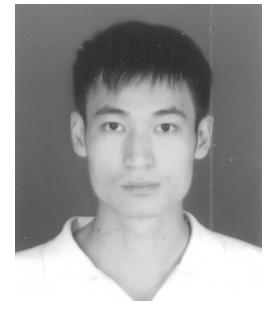

X. L. Zhang was born in Sichuan, China, in 1986. He received the bachelor degree in microelectronics from University of Electronic Science and Technology, China, in 2010. He is currently pursuing a master's degree at the UESTC. His research direction is CMOS RF monolithic circuit design, with a focus on RF receivers and transmitters.

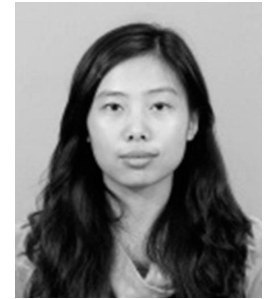

Y. Peng was born in Sichuan, China, in 1988. She received the bachelor degree in microelectronics from University of Electronic Science and Technology, China, in 2011. She is presently pursuing a master's degree at the UESTC. Her research interests are analog RF circuit design for multi-standard radios and RF-receiver applications, in advanced CMOS technology. 